\title{
A Newly Defined Pyroptosis-Related Gene Signature for the Prognosis of Bladder Cancer
}

\author{
Weikang Chen (1D) ${ }^{1, *}$ \\ Wenhao Zhang ${ }^{2,3, *}$ \\ Tao Zhou',* \\ Jian Cai ${ }^{1}$ \\ Zhixian Yu' \\ Zhigang $\mathrm{Wu}^{\prime}$
}

'Department of Urology, The First Affiliated Hospital of Wenzhou Medical University, Wenzhou, Zhejiang, 325000,

People's Republic of China; ${ }^{2}$ The Fourth School of Clinical Medicine, Zhejiang

Chinese Medical University, Hangzhou, Zhejiang, 325000, People's Republic of

China; ${ }^{3}$ Department of Urology, Affiliated Hangzhou First People's Hospital,

Zhejiang University School of Medicine, Hangzhou, Zhejiang, 325000, People's

Republic of China

*These authors contributed equally to this work
Correspondence: Zhixian Yu; Zhigang Wu Department of Urology, The First Affiliated Hospital of Wenzhou Medical University, No. 2, Fuxue Road Lucheng District, Wenzhou, Zhejiang, 325000,

People's Republic of China Email yuzx515@I63.com; andrologywzg@wmu.edu.cn
Background: Bladder cancer (BC), as the most common urinary system tumor type and the main cause of tumor-related death, has an unsatisfactory prognosis. In recent years, related literature has proposed that cell pyroptosis is an inflammatory form of programmed cell death. However, in BC, the relationship between the expression of pyroptosis-related genes and the prognosis has not been elucidated.

Methods: We got the RNA sequencing data from TCGA and GEO datasets. Fifty-two pyroptosis-related genes were extracted for further explore. Then, we compared the gene expression levels between the normal bladder and BC tissues. After that, we develop and validate a pyroptosis-related gene prognostic model and made following functional enrichment analysis and single-sample gene set enrichment analysis of the differentially expressed genes between the high- and low-risk groups.

Results: Twenty-nine differentially expressed genes (DEGs) were found between normal and tumor tissues. Based on the median score calculated by the risk score formula from 8 pyroptosis-related genes, 414 patients were equally divided into low- and high-risk subgroups. The survival probability of $\mathrm{BC}$ patients in the high-risk group was significantly lower than that in the low-risk group $(\mathrm{P}<0.001)$. Through multivariate analysis, our risk score is an independent factor predicting $\mathrm{OS}$ in $\mathrm{BC}$ patients. Gene Ontology (GO) and Kyoto Encyclopedia of Genes and Genomes (KEGG) analysis show that high-risk populations are rich in immune-related genes and have a decreased immune status. All the above results have been externally verified from GEO cohort.

Conclusion: Pyroptosis-related genes are closely related to tumor immunity and are a potential prognostic tool for predicting BCs.

Keywords: pyroptosis, bladder cancer, immune, prognosis, TCGA

\section{Introduction}

Bladder cancer (BC), as the most Common urinary system tumor type and the main cause of tumor-related death, causes 165 thousand deaths every year and leads to high morbidity and mortality in China. ${ }^{1}$ Kinds of intrinsic and extrinsic factors could affect the occurrence and progression of BC (ethnic group, geographical area, treatment modalities, cell type of the bladder cancer, etc). The most common risk factors contain effects of environmental carcinogens, occupational exposure and smoking. ${ }^{2}$ Although a large percentage of $\mathrm{BC}$ is non-muscle invasive bladder cancer (NMIBC) at the first diagnosis, the percent of reoccurrence is almost $30 \%$, needing transurethral resection of bladder tumor for a second time. ${ }^{3}$ A big part of NMIBC patients will finally change into invasive disease. ${ }^{4}$ Neoadjuvant chemotherapy with following radical cystectomy is a standard treatment for muscle invasive bladder cancer (MIBC). However, the OS 
rate of 5 years is unsatisfactory (38.6\%) among cT3-4aN0 patients after above treatments. ${ }^{5}$ In view of the limitations of $\mathrm{BC}$ therapy, new therapeutic targets are needed to improve the survival outcome of $\mathrm{BC}$; therefore, there is an urgent need for reliable new prognostic models to make targeted therapy more flexible.

Pyroptosis, as a new form of programmed cell death, is known as cellular inflammatory necrosis. ${ }^{6,7}$ The gasdermin family is the main executor of pyroptosis. ${ }^{8-12}$ Pyroptosis was firstly found to be an important mechanism for combating infection. Afterwards, more and more researches found that it was related to the occurrence and development of tumors. It has been published that proinflammatory cytokines, gasdermin proteins, and inflammatory vesicles, which are significant components of pyroptosis, are related with invasion, oncogenesis, and metastasis. ${ }^{13}$ Dupaul-Chicoine et $\mathrm{al}^{13}$ found that the transgenic mice knocked out inflammatory vesiclerelated genes (CASP1 and NLRP3) were more probably to develop colon cancers. Besides, the process of pyroptosis always be accompanied by inflammatory response and immune system activation, which is unlike apoptosis. ${ }^{14}$ Some studies have found that the potent proinflammatory effect of pyroptosis plays an important role in the regulation of tumor immune microenvironment. In addition, some studies reported that pyroptosis also plays an important role in the activity of $\mathrm{CD} 8+\mathrm{T}$ lymphocytes ${ }^{15}$ and anti-tumor function of NK cells. ${ }^{16}$

Taking into account the existing research results, we found that pyroptosis plays a vital role in tumor and anti-tumor process. However, its specific functions in $\mathrm{BC}$ have been less studied. Therefore, we conducted this study in order to clarify the expression levels of pyroptosis-related genes between normal and bladder tumor tissues, explore the potential prognostic value of these genes, and try to discover the correlation between them and the tumor immune microenvironment.

\section{Materials and Methods}

\section{Datasets}

The RNA sequencing (RNA-seq) data and clinical information of $414 \mathrm{BC}$ patients and 19 normal human bladder samples were downloaded from the TCGA database on 29 July 2021 (https://portal.gdc.cancer.gov/repository). We obtained the RNA-seq data and the clinical information from TCGA database and GEO database (https://www. ncbi.nlm.nih.gov/geo/, ID: GSE31684).

\section{Distinguish the Differentially Expressed Genes Related to Pyroptosis}

Fifty-two pyroptosis-related genes from prior reviews were extracted, ${ }^{17-20}$ and the list of full names is presented in the supplementary file (Table S1). We used "limma" package to distinguish DEGs with a $\mathrm{P}$ value $<0.05$. Then, we constructed a DEGs-related PPI network by Search Tool (STRING, version 11.5, https://string-db.org/) for the Retrieval of Interacting Genes.

\section{Development and Validation of the Prognostic Model Related to Pyroptosis Gene}

We further took Cox regression analysis to excavate the relationships between each gene and survival status in the TCGA group. The cut-off P-value was 0.2 , and 9 survivalrelated genes were selected. The LASSO Cox regression model was used by R package "glmnet" to improve the prognostic model. Finally, the eight genes and their coefficients were gotten. The risk score was calculated by "scale" function in R in the TCGA data. Then, The BC patients were divided into high- and low-risk groups according to the median risk score, and the KaplanMeier analysis of OS time, 1,3,5-year ROC curve, PCA and t-SNE was performed. Distribution and survival status of patients was showed by the "bioRiskPlot" function based on the risk score. For the validation studies, the same procedure operates in GEO data (GSE31684).

\section{Independent Prognostic Analysis of the Risk Score}

We got the clinical information (age, gender, stage, grade, TNM) of patients in the TCGA group. Then, we employed Univariate and multivariable Cox regression models for the analysis. Due to the lack of above clinical information in GEO data, Univariate and multivariable Cox regression analysis was not continued.

\section{Functional Enrichment Analysis of the DEGs}

BC patients were divided into two cohorts by the median risk score. The DEGs between the high- and low-risk groups were filtered (FDR $<0.05$ and $|\log 2 \mathrm{FC}| \geq 1$ ). Based on these DEGs, GO and KEGG analyses were performed by applying the "clusterProfiler" package. The "gsva" package was utilized to conduct the ssGSEA to 
calculate the scores of infiltrating immune cells and to evaluate the activity of immune-related pathways.

\section{Statistical Analysis}

Single-factor analysis of variance was used for comparing the gene expression levels, while the Pearson chi-square test was applied for categorical variables. The KaplanMeier method with a two-sided Log rank test, univariate and multivariate Cox regression models was employed for comparing the OS of patients between subgroups and assessing the independent prognostic value of the risk model. The Mann-Whitney test was used when comparing the immune cell infiltration and pathway activation between the two cohorts. All statistical analyses were accomplished with R software (v4.1.0).

\section{Results}

\section{Identification of DEGs Between Normal and Tumor Tissues}

The 53 gene expression levels related to pyroptosis were compared in TCGA data from 414 tumor and 19 normal tissues, and finally we found 29 DEGs $(\mathrm{P}<0.05)$. Among these genes, 6 genes (CHMP7, NLRP1, CHMP3, NLRP3, IL6 and ELANE) were downregulated, and 23 genes (CASP5, AIM2, NLRP2, IL1A, NLRP7, CHMP4C, CASP6, GSDMB, BAX, TP63, PYCARD, BAK1, CASP3, CASP8, GSDMD, TP53, PLCG1, GPX4, CHMP2A, HMGB1, CHMP4B, CHMP4A and CYCS) were enriched in the tumor cohort. The heatmaps of RNA levels and the PPI analysis are shown in Figures $1 \mathrm{~A}$ and $\mathrm{B}$. The minimum required interaction score was 0.4, and we determined that AIM2, BAK1, BAX, CASP3, CASP5, CASP6, CASP8, CHMP2A, CHMP3, CYCS, ELANE, GSDMD, HMGB1, IL1A and IL6 were hub genes. The correlation network including all pyroptosis-related genes is showed in Figure 1C.

\section{Tumor Classification Based on the DEGs}

We used a consensus clustering analysis with 414 BC patients in the TCGA cohort for exploring the connections and we found that the intragroup correlations were the highest and the inter-group correlations were low when $\mathrm{k}=2$ (Figure 2A). The gene expression and the clinical information including age ( $\leq 65$ or $>65$ years), gender (female or male), the degree of tumor differentiation (high grade, low grade or unknown), $\mathrm{T}$ stage (T0,1,2,3,4 or unknown), $\mathrm{N}$ stage (N0,1, 2, 3 or unknown) and $\mathrm{M}$ stage (M0,1 or unknown) were showed in a heatmap, and we found there was little differences in clinical features, except gender (Figure 2B). The OS was also compared between the two cohorts, but no significant differences were discovered $(P=0.293$, Figure 2C).

\section{Development of a Prognostic Gene Model in the TCGA Cohort}

We used univariate Cox regression analysis for survival-related genes. The 9 genes (BAK1, CASP1, GZMB, IRF1, TP63, AIM2, NOD 2, TNF and GZMA) were selected for further analysis $(\mathrm{P}<0.2)$ (Figure 3A). After LASSO Cox regression analysis, we constructed an 8-gene signature (Figure $3 \mathrm{~B}$ and $\mathrm{C}$ ). The result was showed as follows: risk score = $(-0.010 *$ BAK1 $\quad$ exp. $)+(-0.012 *$ GZMB $\quad$ exp. $)+$ $(-0.073 *$ IRF1 exp. $+(-0.063 *$ TP63 exp. $)$ $+(-0.033 *$ AIM2 exp. $)+(0.233 *$ NOD2 exp. $)+$ $(-0.114 * \mathrm{TNF} \quad$ exp. $)+(-0.032 *$ GZMA exp. $) .414$ patients were equally divided into high-risk and lowrisk subgroups, and a significant difference of OS was detected between these two groups $(\mathrm{P}<0.001$, Figure 3D). Time-dependent ROC analysis was used to evaluate the specificity and sensitivity of the prognosis model, and the AUC was 0.631, 0.626, and 0.631 for 1-, 3- and 5-year survival (Figure 3E). High-risk group had more patient deaths and shorter survival time than low-risk group (Figure $3 F$ and $G$ ). The PCA and t-SNE (Figure $3 \mathrm{H}$ and $\mathrm{I}$ ) showed that patients were well divided into two clusters.

\section{External Validation of the Risk Signature}

Ninety-three BC patients from GEO cohort (GSE31684) were used as the validation cohort. Forty-nine patients in the GEO cohort were separated into the low-risk group, and 44 patients were separated into the high-risk group by the median risk score in the TCGA cohort. A significant difference in OS was found between the high- and low-risk groups $(\mathrm{P}=0.012$, Figure 4A). Time-dependent ROC analysis was used to assess the sensitivity and specificity of the prognosis model, and we detected that the area under the AUC was 0.562, 0.578 and 0.653 for 1-, 3- and 5-year survival (Figure 4B).

High-risk group had more patient deaths and shorter survival time than low-risk group (Figure 4C and D). The PCA and t-SNE (Figure 4E and F) showed that patients were well divided into two clusters. 


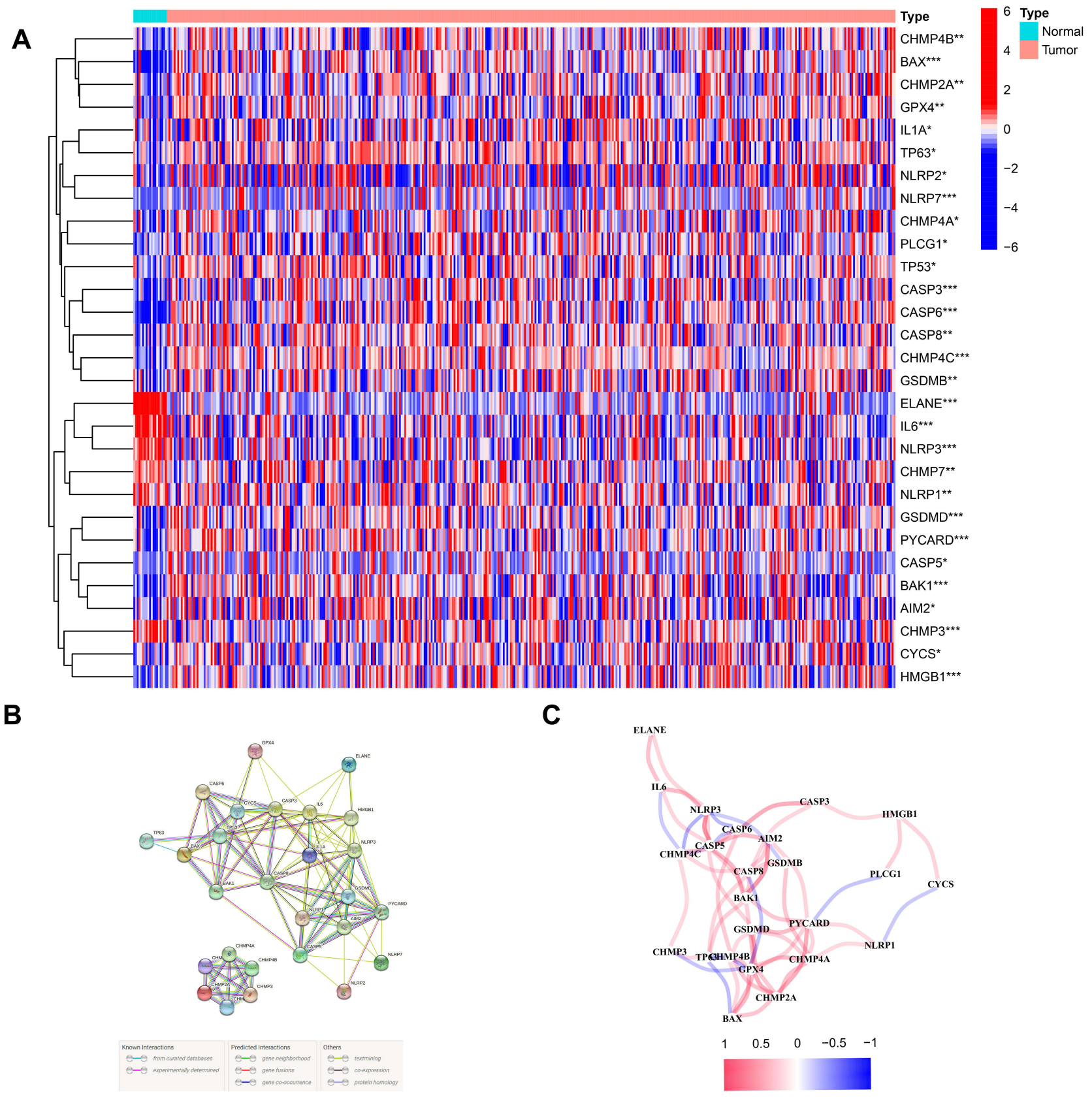

Figure I Expressions of the 29 pyroptosis-related genes and the interactions among them. (A) Heatmap of the pyroptosis-related genes between the normal and the tumor tissues. (B) PPI network showing the interactions of the pyroptosis-related genes (interaction score $=0.4$ ). (C) The correlation network of the pyroptosis-related genes. $\mathrm{P}$ values were showed as: $* * \mathrm{P}<0.01 ; * * * \mathrm{P}<0.001$.

Independent Prognostic Value of the Risk Model

Univariate and multivariable Cox regression analyses were used to find the independent prognostic factor, and finally indicated that the risk score was an independent factor in both univariate and multivariate Cox regression analysis $(\mathrm{HR}=5.214,95 \% \mathrm{CI}: 1.993-13.644$, Figure 5A; HR = 3.629, 95\% CI: 1.334-9.873, Figure 5B). Besides, we created a heatmap of clinical features for the TCGA cohort and detected that the $M$ stage was significantly different between the two subgroups $(\mathrm{P}<0.01$, Figure 5E).

\section{Functional Analyses Based on the Risk Model}

One hundred and fourteen DEGs between the high and low groups in the TCGA cohort were identified after the use of "limma" R package. Among these genes, 20 genes were up-regulated in the high-risk group, and 94 genes 
A

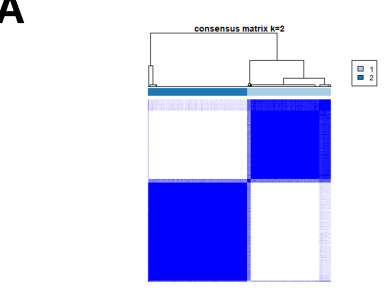

C

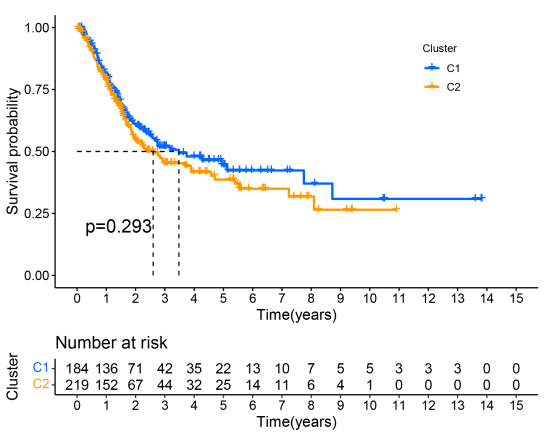

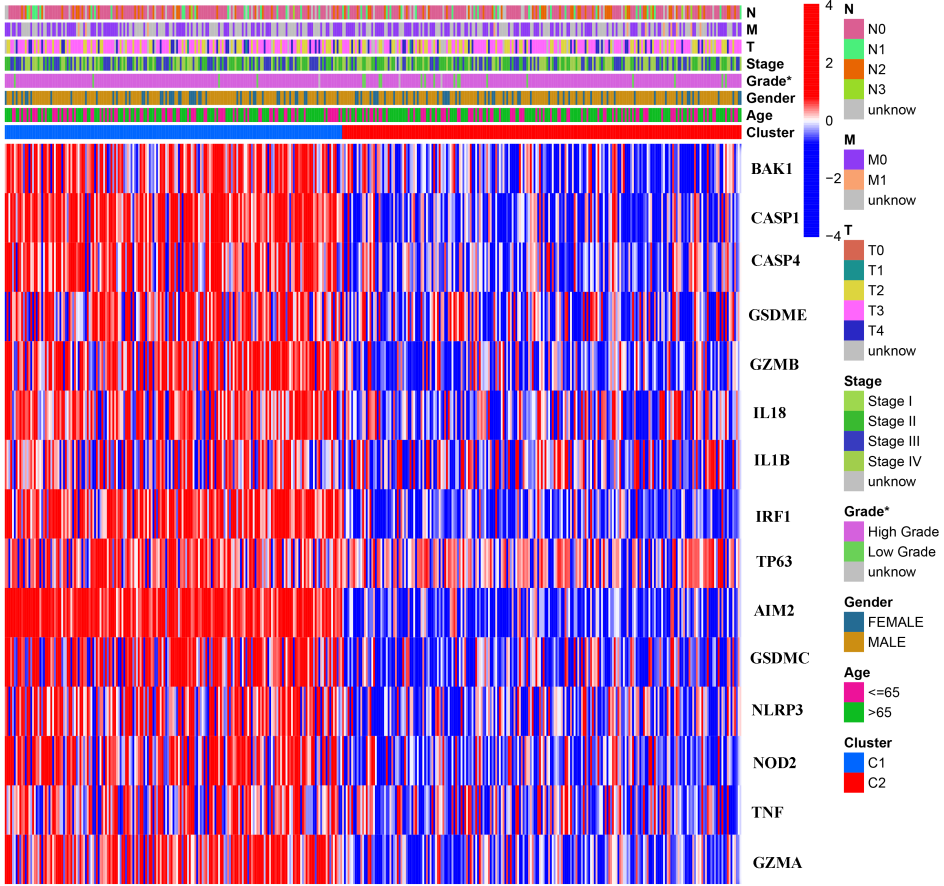

Figure 2 Tumor classification based on the pyroptosis-related DEGs. (A) 4I4BC patients were divided into two clusters by the consensus clustering matrix ( $\mathrm{k}=2$ ). (B) Heatmap and the clinicopathologic characters of the two clusters classified by these DEGs. (C) Kaplan-Meier curves for the OS of these two clusters.

were down-regulated (showed in the supplementary file (Table S2)). The results of GO enrichment analysis and Kyoto KEGG pathway analysis indicated that the DEGs play an important role in the immune response, response to virus and antigen processing and presentation (Figure 6A and $\mathrm{B})$.

\section{Comparison of the Immune Activity Between Subgroups}

We employed the ssGSEA by comparing the enrichment scores of the activity of 13 immune-related pathways and 16 types of immune cells between the different groups in TCGA and GEO groups. Except for mast cells, the other immune cells showed lower levels of infiltration in the high-risk group in the TCGA cohort and GEO cohorts (Figures 7A and $\underline{\mathrm{C}}$ ). In the TCGA and GEO cohorts (Figures 7B and $\underline{D}$ ), the high-risk subgroup generally had lower activity of all immune pathways, except the type-2 IFN response pathway.

\section{Discussion}

In the study, we researched the mRNA levels of 53 recently known genes related to pyroptosis in normal bladder and $\mathrm{BC}$ tissues and discovered that more than half of these genes were differentially expressed. Then, we used the consensus clustering analysis to divide $\mathrm{BC}$ patients into two clusters based on the DEGs, but there was no significant difference in the survival. In order to further explore the prognostic role of these genes, we created an 8-gene risk signature. The following functional analyses suggested that the DEGs between the high- and low-risk groups were bound up with immune response. We compared the immune cells and immune pathways in the low- and high-risk groups and discovered that the highrisk group had less infiltrating immune cells and less active immune-related pathways.

Pyroptosis is a new type of programmed death, which has now been found to have an important double-sided role in the development of tumors. On the one hand, it can stimulate normal cells to transform into tumor cells by releasing a large number of inflammatory factors. On the other hand, the process of pyroptosis could be a potential therapeutic target. In patients with bladder cancer, the correlation between these pyroptosis-genes and the relationship between them and the survival is still unclear. The study constructed a signature featuring 8 pyroptosisrelated genes (BAK1, GZMB, IRF1, TP63, AIM2, NOD 2, TNF and GZMA) and found it could forecast survival in $\mathrm{BC}$ patients.

BAK1 is initially established as an ultimate death effector of apoptosis triggering cues. One study 
A

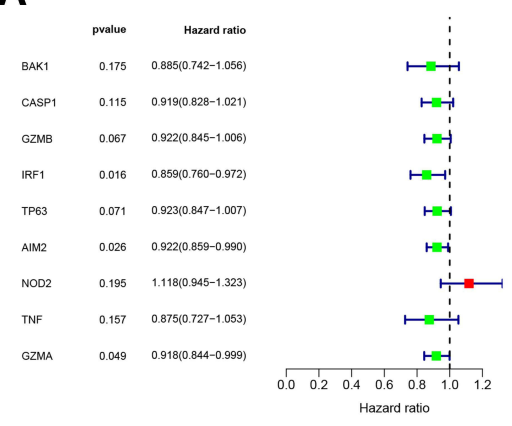

D
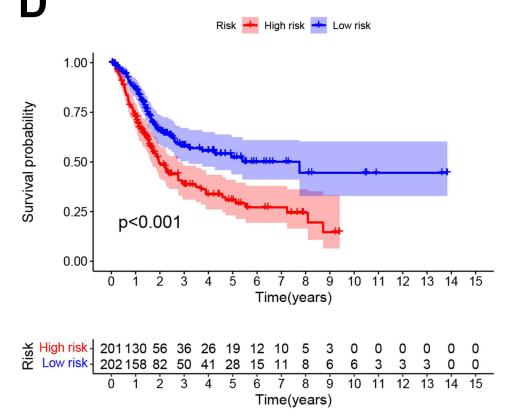

G

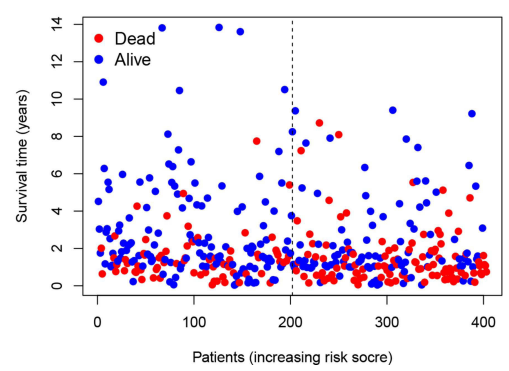

B

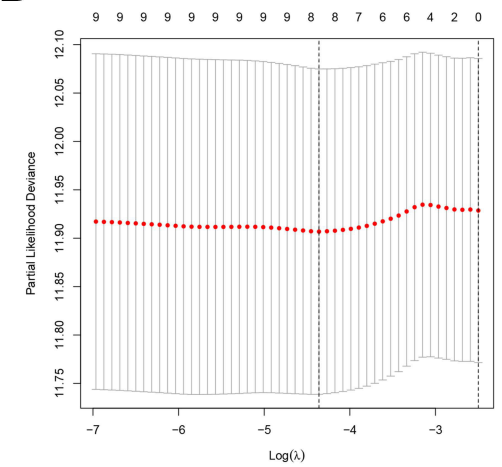

E
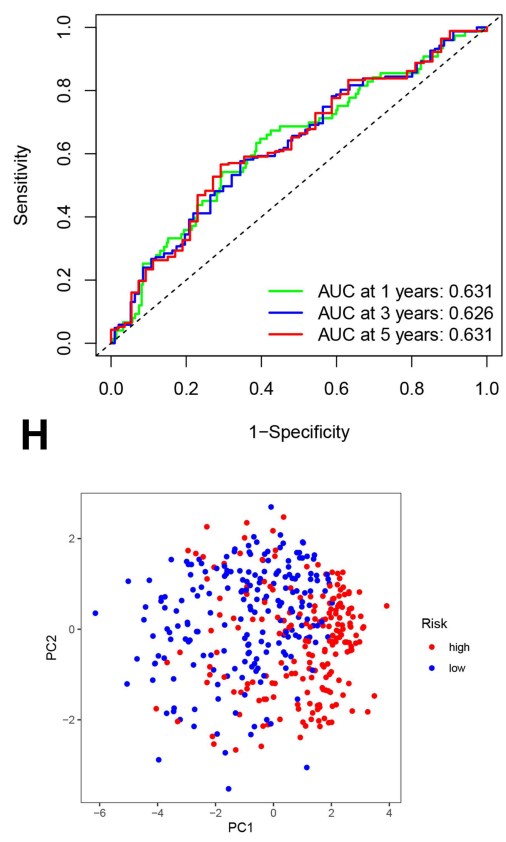

C

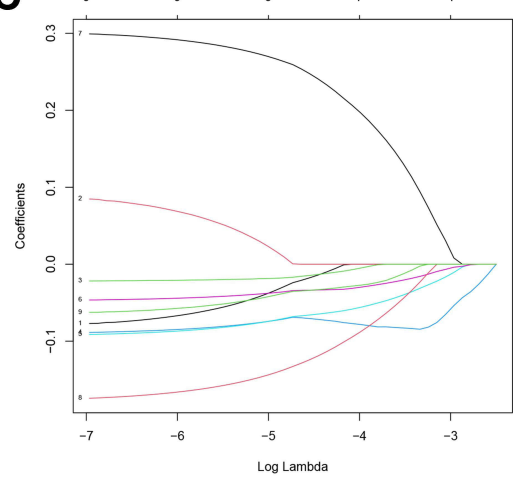

$\mathbf{F}$

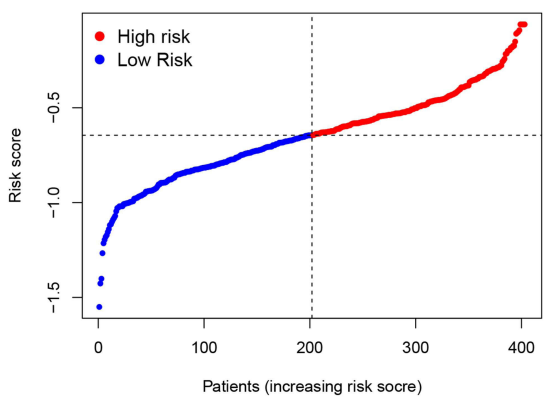

I

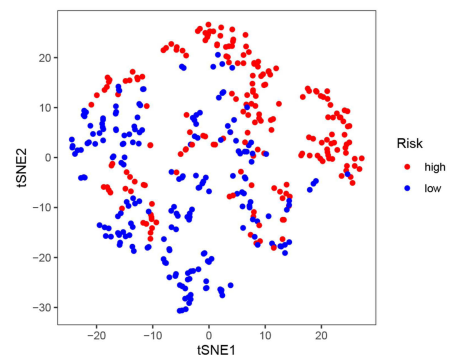

Figure 3 Construction of risk signature in the TCGA cohort. (A) Univariate cox regression analysis of OS for selected pyroptosis-related gene with P < 0.2. (B) LASSO regression of the 9 OS-related genes. (C) Cross-validation for tuning the parameter selection in the LASSO regression. (D) Kaplan-Meier OS curves of patients in the highand low-risk groups. (E) ROC curves demonstrated the predictive efficiency of the risk score. (F) Distribution of patients based on the risk score. (G) The survival status for each patient. (H) PCA plot for BCs based on the risk score. (I) t-SNE plot for BCs based on the risk score.

demonstrated that BAK1 could be directly targeted by miR-125b to mediate breast cancer progression. ${ }^{21}$ It showed that BAK1 could significantly enhance Taxolinduced cytotoxicity and apoptosis. Similarly, another study indicated that BAK1 suppression caused by specific miRNA could lead to androgen-independent growth of prostate cancer cells. ${ }^{22}$ However, BAK1 is regarded as an unfavorable prognostic factor in some studies for Hepatocellular Carcinoma and Head and Neck Squamous Cell Carcinoma. ${ }^{23,24}$ In the study, BAK1 seems to be a cancer-promoting gene, because its expression in tumor tissues is significantly higher than that in normal tissues; however, it helps to prolong patient survival for that it is enriched in the low-risk group. Given that the data provided by $\mathrm{BC}$ is limited and the results of different tumors are often contradictory, our results on BAK1 provide some insights for further research.

Granzyme B (GZMB) is normally produced as a toxic granule-secreted enzyme with killing activity in the tumor microenvironment by natural killer cells and cytotoxic $\mathrm{T}$ lymphocytes. ${ }^{25,26}$ But some reports have reported that low GZMB expression may be related to early CRC metastasis. ${ }^{27}$ Besides, GZMB can cut downstream caspase-3 and bid to activate them with hydrolytic activity as a serine protease, resulting in apoptosis of target-cells. ${ }^{28,29}$ Moreover, a study detected that, in Colorectal cancer cells, LINC02474 was associated with the transcriptional repression of GZMB determined by 
A
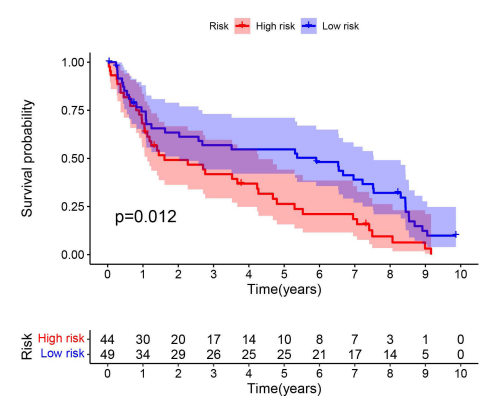

D

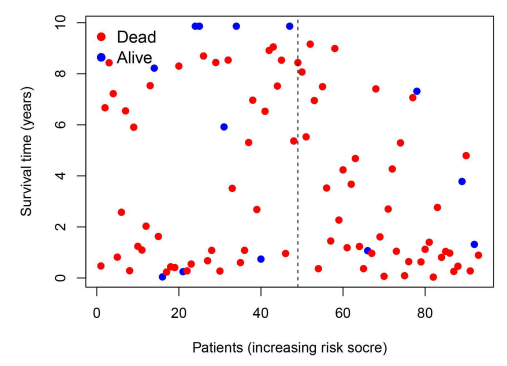

B

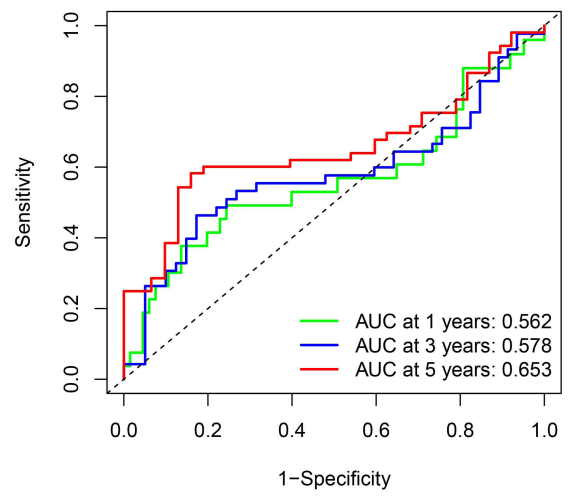

E

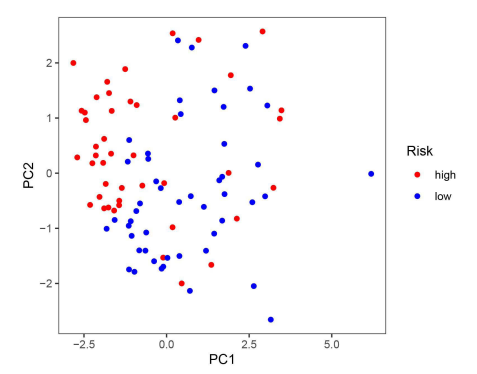

C

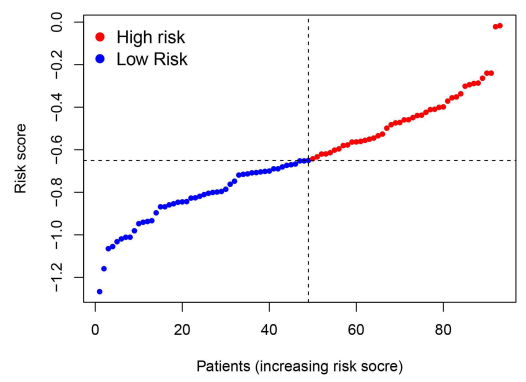

$\mathbf{F}$

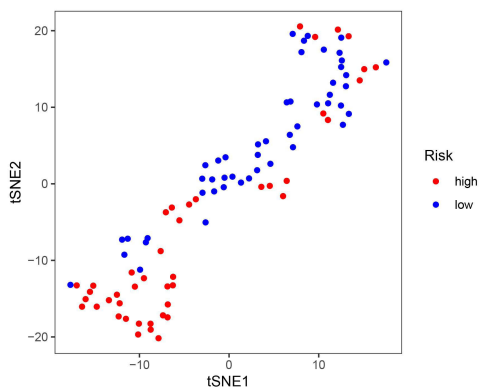

Figure 4 Validation of the risk model in the GEO cohort. (A) Kaplan-Meier curves for comparison of the OS between low- and high-risk groups. (B) Time-dependent ROC curves for BCs. (C) Distribution of patients in the GEO cohort based on the median risk score in the TCGA cohort. (D) The survival status for each patient (low-risk population: on the left side of the dotted line; high-risk population: on the right side of the dotted line). (E) PCA plot for BCs. (F) t-SNE plot for BCs based on the risk score.

A

$$
\begin{array}{lrr}
\text { A } & \text { pvalue } & \text { Hazard ratio } \\
\text { Age } & 0.053 & 1.025(1.000-1.051) \\
\text { Gender } & 0.099 & 0.648(0.387-1.085) \\
\text { Grade } & 0.191 & 0.266(0.037-1.935) \\
\text { T } & 0.008 & 1.627(1.134-2.336) \\
\text { M } & 0.051 & 2.499(0.998-6.260) \\
\text { N } & <0.001 & 1.550(1.204-1.996) \\
\text { riskScore } & <0.001 & 5.214(1.993-13.644)
\end{array}
$$

\section{B}
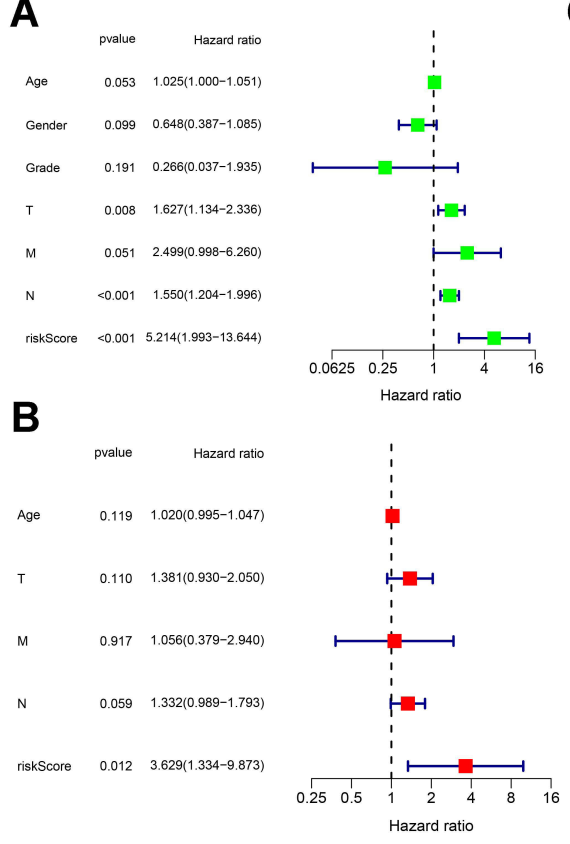

C

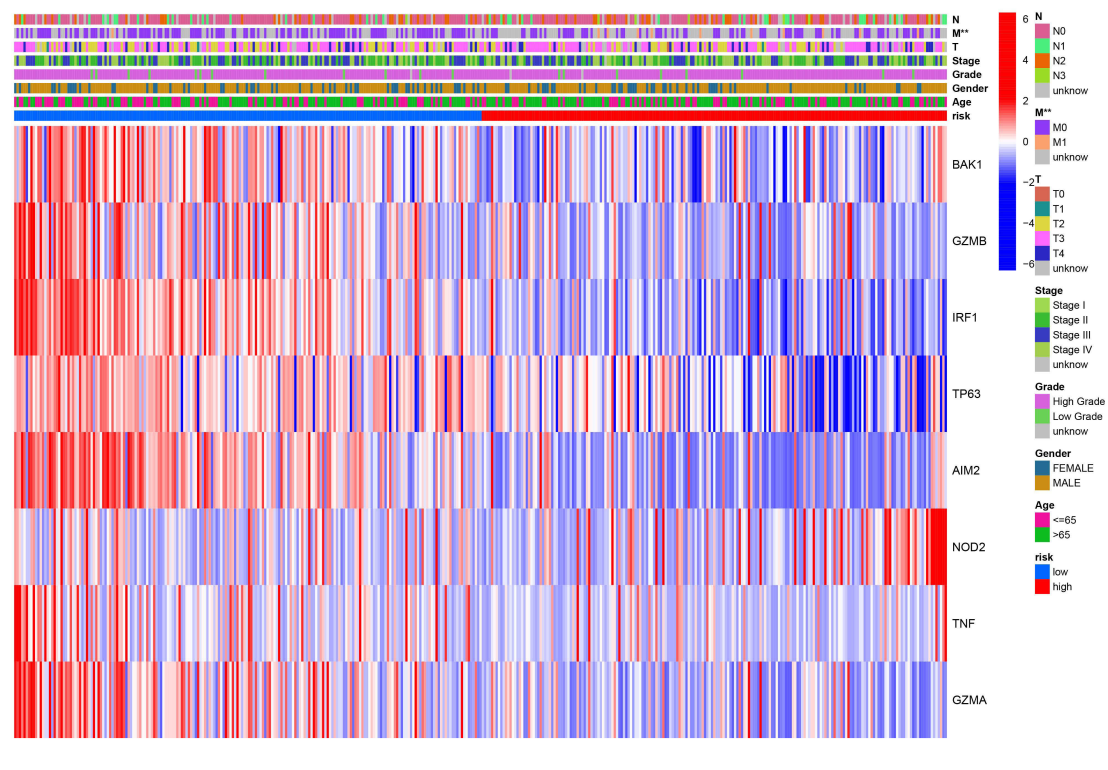

Figure 5 Univariate and multivariate Cox regression analyses for the risk score. (A) Univariate analysis for the TCGA cohort. (B) Multivariate analysis for the TCGA cohort. (C) Heatmap for the connections between clinicopathologic features and the risk groups. P values were showed as: **P $<0.01$. 
A

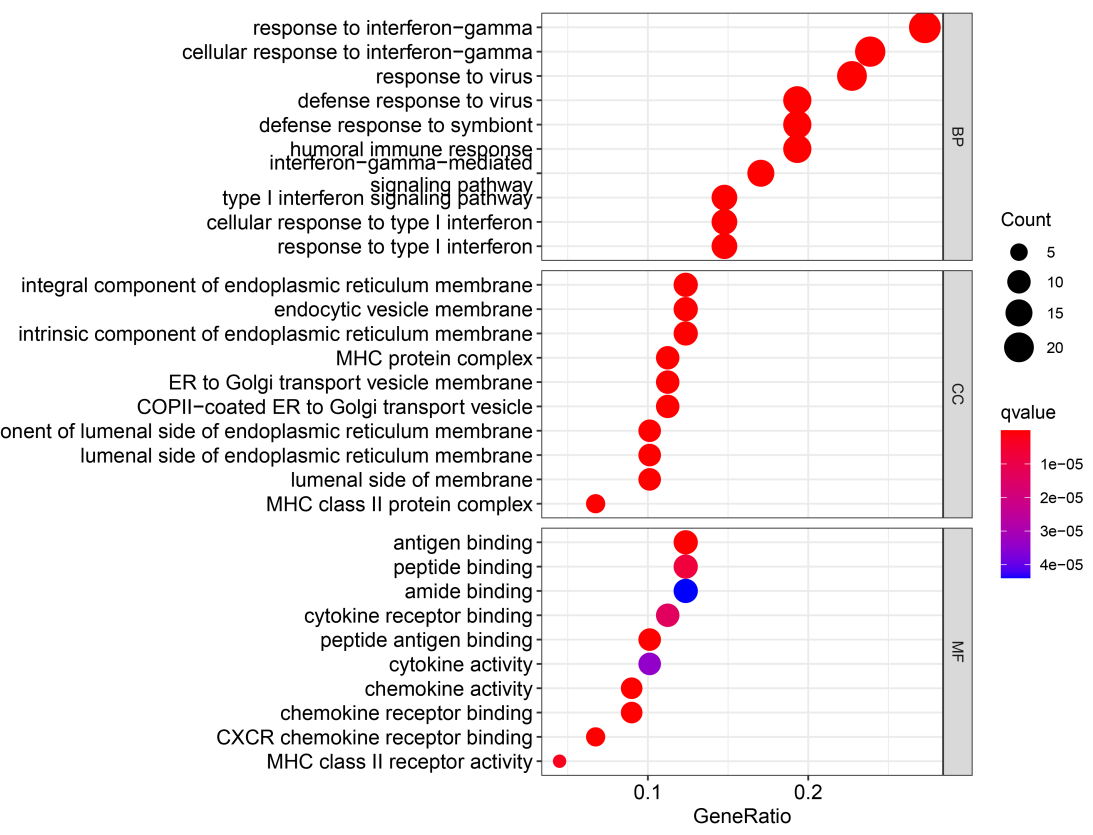

B

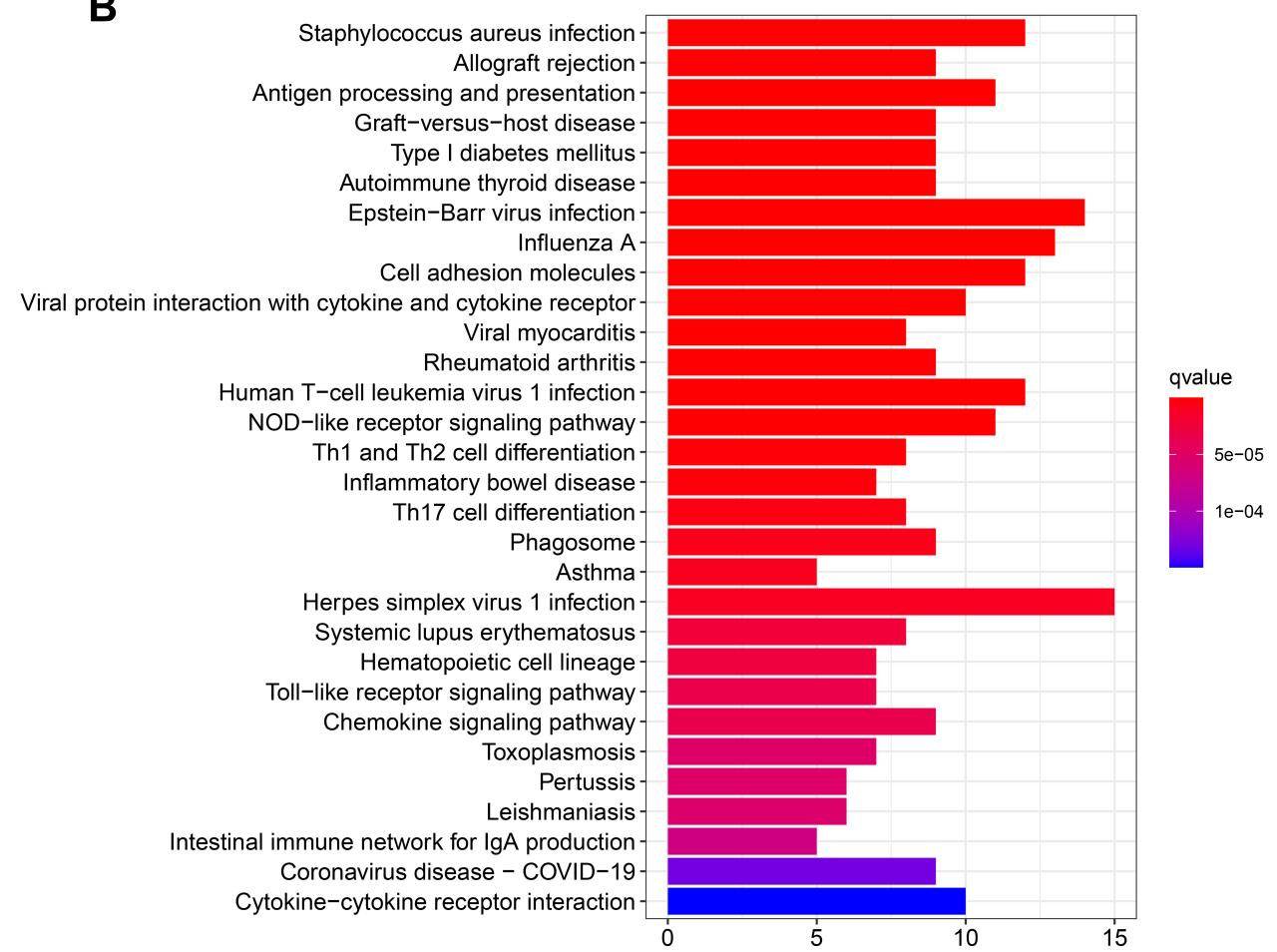

Figure 6 Functional analysis based on the DEGs between the two-risk groups in the TCGA cohort. (A) Bubble graph for GO enrichment (the bigger bubble means the more genes enriched, and the increasing depth of red means the differences were more obvious; q-value: the adjusted p-value). (B) Barplot graph for KEGG pathways (the longer bar means the more genes enriched, and the increasing depth of red means the differences were more obvious).

the dual-luciferase reporter assay and then interference of GZMB could increase the metastatic abilities of CRC cells by reducing apoptosis. In our study, we found that the GZMB gene is enriched in the low-risk group, which is an indicator of good prognosis. However, more studies are demanded to determine how this gene is involved in tumor suppression in the pyroptosis program.

Unlike GZMB, the actual function and mechanism of action of GZMA (another member of the granzyme family) remain unclear, likely because of the lack of 

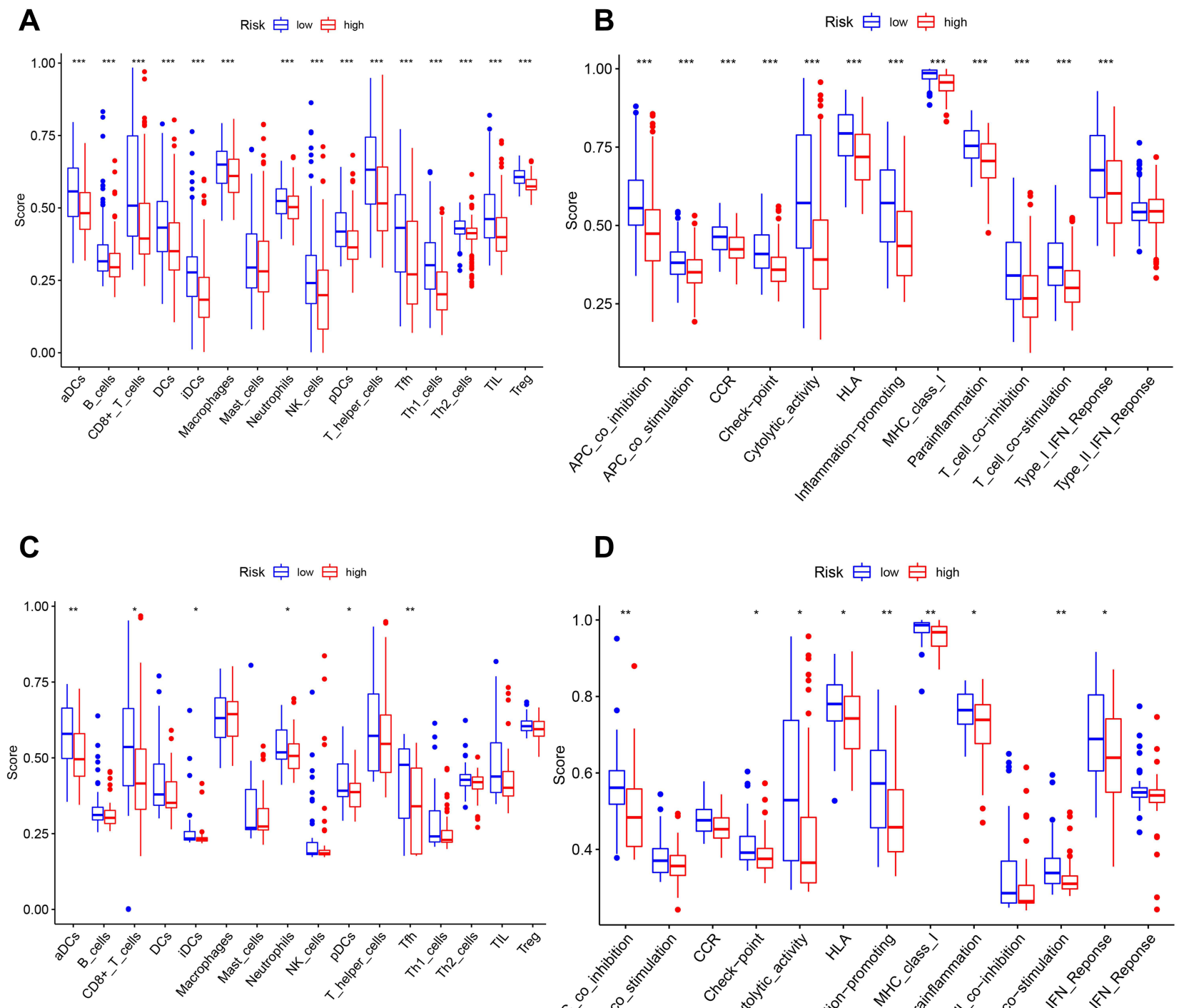

D

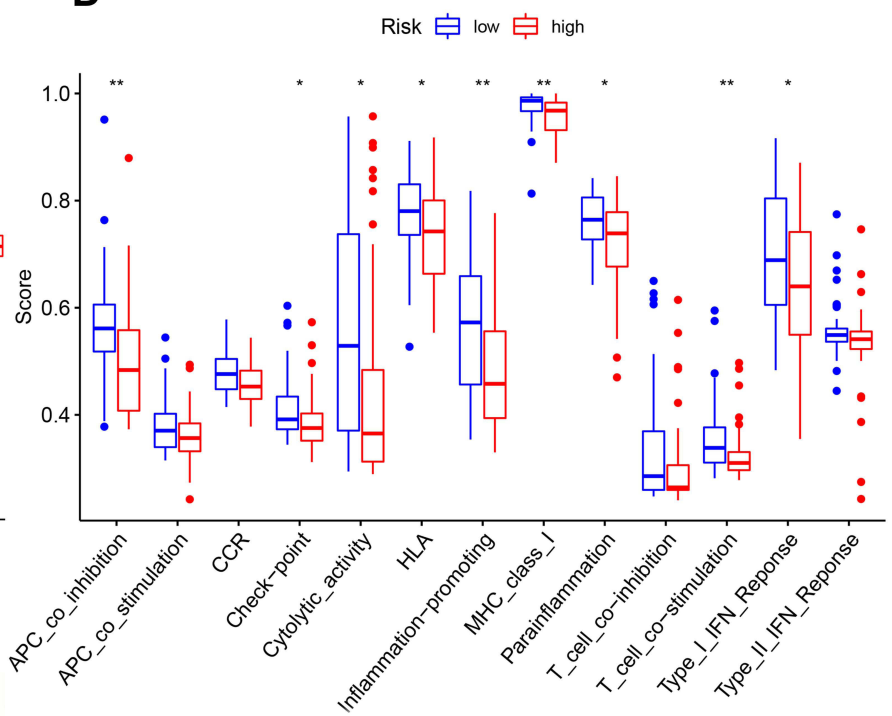

Figure 7 Comparison of the ssGSEA scores for immune cells and immune pathways. (A-D) Comparison of the tumor immunity between low-and high-risk group in the TCGA and GEO cohort. *P < 0.05; **P< 0.01 ; ***P $<0.001$.

sufficiently relevant experimental systems. A recent study discovered that GZMA from cytotoxic lymphocytes cleaves and activates GSDMB to induce target cell pyroptosis. ${ }^{30}$ This immune effector mechanism promotes CTL-mediated tumor clearance in mice. We found that the high expression of GZMA is associated with better survival outcomes. Therefore, whether its mechanism in $\mathrm{BC}$ is the same as the above research is worthy of our follow-up study.

AIM2 could use ASC-mediated junctional proteins to activate CASP-1 and to promote pyroptosis. ${ }^{31}$ AIM2 was considered to be a tumor suppressor for that its mutation or inactivation was discovered in kinds of tumors, including gastric, endometrial and colon cancers, but it was different in non-small-cell lung, nasopharyngeal, and oral cancer. ${ }^{32}$ Therefore, AIM2 may play a different role in different kinds of cancers. In this study, AIM 2 is likely a cancerpromoting gene and may be associated with a good prognosis. We hope that this discovery can pave the way for subsequent research on bladder cancer.

Interferon regulatory factor 1 (IRF1), as a transcription factor induced in response to interferons, ${ }^{33}$ plays crucial roles in various physiological and pathological processes, including inflammation, microbial infection, tumor immune surveillance, and innate and adaptive immune responses. ${ }^{34,35}$ IRF1 controls the gene expression of guanylate-binding 
proteins, the inducible nitric oxide synthase, and caspase- 1 (CASP1), ${ }^{34,36}$ which have been implicated in various inflammatory diseases. Besides, IRF1 was reported that it could prevent tumorigenesis in a spontaneous mouse model of colorectal cancer due to defective pyroptosis, apoptosis, and necroptosis. ${ }^{37}$ More studies are needed focusing on how/ whether IRF1 takes part in tumor suppression and pyroptosis.

TP63 belongs to the TP53 family of tumor suppressor genes, ${ }^{38}$ and these genes induce cell cycle arrest and apoptosis. $^{39,41}$ In particular, TP63, which encodes 2 subtypes of TAP63 and carcinogenic deltaNp63 subtypes, ${ }^{40}$ 14 has been involved in many cancers such as bladder, uterine, and breast cancer, as well as squamous cell carcinoma of the head and neck. ${ }^{42-45}$ Somerville et al $^{46}$ found that the deltaNp63 subtype-driven enhancer reprogramming enhances tumor growth and the invasive ability of cells, promoting epithelial differentiation and metastasis of cancer cells. Moreover, they showed that the continuous expression of TP63 is important for the growth of cancer epithelial cells. However, the correlation between TP63 and cell pyroptosis or tumor prognosis is less researched, and it is worthy of our continued exploration.

The TNF family, as one of the best-studied protein families, includes the 29 TNF receptor superfamily and 19 TNF ligands superfamily. ${ }^{47}$ Some studies have found that TNF can through TNFR1 and TNFR2 and then activate immune cells, as an inflammatory regulator. ${ }^{48}$ Moreover, some studies have detected that many cancers are closely associated with the TNF family. ${ }^{49}$ In our study, we found that TNF is associated with the prognosis of patients with bladder cancer, and its high expression means a better prognosis. However, further experimentally studies are needed for the specific mechanism.

NOD2 belongs to the NOD like receptor family and can interact with many proteins and regulate immune responses. A study found that activated NOD2 can recruit receptor-interacting protein 2 , and conducts signal through nuclear factor (NF)- $\mathrm{B}$ and MAPK pathway. ${ }^{50,51}$ Moreover, NOD2 can promote myocardial apoptosis ${ }^{52}$ and promote renal injury in diabetic nephropathy. ${ }^{53}$ A recent study found that LPS can induce Extracellular histone $\mathrm{H} 3$ and then cause pyroptosis via VSIG4/NLRP3 and NOD2 pathway during sepsis. However, the mechanism of tumor-related pyroptosis induced by NOD2 has rarely been studied. Our study found that high expression of NOD2 is associated with a worse prognosis. This may remind us that NOD2 may affect the prognosis of cancer patients by mediating pyroptosis.
In summary, all eight of the above-mentioned pyroptosis-related genes have some positive or negative effects on the prognosis of bladder cancer patients in our study. The interrelationship of these genes in the pyroptosis process is worthy of our further study.

Nowadays, the research on pyroptosis is not thorough enough. Pyroptosis, as one of the processes of programmed death, may co-occur with apoptosis and other mechanisms that promote cell death and interlock with each other. ${ }^{54}$ For example, BAK1 and GZMB are the key regulator in the apoptosis mechanism. Different from apoptosis, pyroptosis could lead to the release of cell contents and then activates a strong inflammatory response, which can also be regarded as an important natural immune response of the body. Through the analysis of patients in different risk groups, we found that DEGs mainly covers cellular immunity and inflammation. Through the following GO and KEGG analysis, we can speculate that pyroptosis is related to the cellular immune microenvironment.

In addition, we compared the levels of key antitumor infiltrating immune cells between high- and low-risk cohorts from TCGA and GEO datasets, and finally found an overall suppression of immune functions in the high-risk group. Previous studies reported that treg cells could suppress antitumor immunity and are associated with poor clinical outcomes. In the study, we found that treg cells are enriched in a low-risk group. The reason for different conclusions may be associated with GZMB. GZMB has been detected to be expressed by human and mouse Treg cells and suppress the immune function and cancer immune-evasion by inhibiting NK and Tc cell responses to control viral-induced lung inflammation or in tumor models. ${ }^{55-58}$ Besides, it may be related to different Treg cell subtypes. ${ }^{59}$ Through these discoveries, the poor survival outcome of highrisk BCs may be generated by decreased levels of antitumor immunity, and the specific mechanism needs our future research.

There are also some limitations with our study. This is a preliminary research for the prognostic value of pyroptosis-related genes, and we hope to provide some theoretical basis for future research. Because of the lack of relevant research, we cannot confirm whether the above regulators play similar roles in pyroptosis pathways in bladder cancer patients, so in-depth researches are needed to confirm this question. In addition, there were potentially important confounders, such as treatment measures, for 
which data were not available in our database. Therefore, we hope that further prospective studies could be conducted to confirm our findings. Last but not least, we believe that the real-life research by our institution would contribute and validate these results in the future.

In conclusions, we discovered that pyroptosis is related to $\mathrm{BC}$ closely, because a big part of the pyroptosis-related genes were differently expressed between normal and $\mathrm{BC}$ tissues. Besides, the score based on 8 pyroptosis-related genes generated from our risk signature was an independent risk factor for predicting OS. The DEGs between the high and low-risk groups were connected to tumor immunity. Our study offers a new gene signature for predicting the prognosis of $\mathrm{BC}$ patients and provides an important foundation for future researches of the relations between pyroptosis-related genes and immunity in BC.

\section{Data Sharing Statement}

The data could be download at (https://portal.gdc.cancer. gov/ and https://www.ncbi.nlm.nih.gov/geo/).

\section{Ethics Approval and Consent to Participate}

Approval for this study was granted by the Ethics Committee of The First Affiliated Hospital of Wenzhou Medical University, Wenzhou, Zhejiang Province, China.

\section{Author Contributions}

All authors contributed to data analysis, drafting or revising the article, gave final approval of the version to be published, agreed to the submitted journal, and agreed to be accountable for all aspects of the work.

\section{Funding}

Supported by Foundation of Zhe'jiang Educational Committee (Y202045482).

\section{Disclosure}

The authors declare no competing interests.

\section{References}

1. Chen W, Zheng R, Baade PD, et al. Cancer statistics in China, 2015. CA Cancer J Clin. 2016;66(2):115-132. doi:10.3322/caac.21338

2. Cumberbatch MG, Cox A, Teare D, Catto JW. Contemporary occupational carcinogen exposure and bladder cancer: a systematic review and meta-analysis. JAMA Oncol. 2015;1:1282-1290.

3. Felsenstein KM, Theodorescu D. Precision medicine for urothelial bladder cancer: update on tumour genomics and immunotherapy. Nat Rev Urol. 2018;15:92-111.
4. Santoni G, Morelli MB, Amantini C, Battelli N. Urinary markers in bladder cancer: an update. Front Oncol. 2018;8:362.

5. Nitta M, Kuroda S, Nagao K, et al. Effect of neoadjuvant chemotherapy in patients undergoing radical cystectomy for muscle-invasive bladder cancer: a retrospective, multi-institutional study. Jpn J Clin Oncol. 2019;50(1):73-79.

6. Kovacs SB, Miao EA. Gasdermins: effectors of pyroptosis. Trends Cell Biol. 2017;27:673-684.

7. Miao EA, Rajan JV, Aderem A. Caspase-1-induced pyroptotic cell death. Immunol Rev. 2011;243:206-214.

8. Broz P, Pelegrín P, Shao F. The gasdermins, a protein family executing cell death and inflammation. Nat Rev Immunol. 2020;20:3.

9. Ding J, Wang K, Liu W, et al. Pore-forming activity and structural autoinhibition of the gasdermin family. Nature. 2016;535:111-116.

10. Feng S, Fox D, Man SM. Mechanisms of gasdermin family members in inflammasome signaling and cell death. $J$ Mol Biol. 2018;430:3068-3080.

11. Zhang Y, Chen X, Gueydan C, Han J. Plasma membrane changes during programmed cell deaths. Cell Res. 2018;28:9-21.

12. Frank D, Vince JE. Pyroptosis versus necroptosis: similarities, differences, and crosstalk. Cell Death Differ. 2019;26:99-114.

13. Dupaul-Chicoine J, Yeretssian G, Doiron K, et al. Control of intestinal homeostasis, colitis, and colitis-associated colorectal cancer by the inflammatory caspases. Immunity. 2010;32:367-378.

14. Tang R, Xu J, Zhang B, et al. Ferroptosis, necroptosis, and pyroptosis in anticancer immunity. J Hematol Oncol. 2020;13:110.

15. Xi G, Gao J, Wan B, et al. GSDMD is required for effector CD8 $\mathrm{T}$ cell responses to lung cancer cells. Int Immunopharmacol. 2019;74:105713.

16. Zhang Z, Zhang Y, Xia S, et al. Gasdermin E suppresses tumour growth by activating anti-tumour immunity. Nature. 2020;579:415-420.

17. Karki R, Kanneganti TD. Diverging inflammasome signals in tumorigenesis and potential targeting. Nat Rev Cancer. 2019;19:197-214.

18. Xia X, Wang X, Cheng Z, et al. The role of pyroptosis in cancer: ProCancer or Pro-"Host"? Cell Death Dis. 2019;10:650.

19. Wang B, Yin Q. AIM2 inflammasome activation and regulation: a structural perspective. J Struct Biol. 2017;200:279-282.

20. Man SM, Kanneganti TD. Regulation of inflammasome activation. Immunol Rev. 2015;265:6-21.

21. Zhou M, Liu Z, Zhao Y, et al. MicroRNA-125b confers the resistance of breast cancer cells to paclitaxel through suppression of proapoptotic Bcl-2 antagonist killer 1 (Bak1) expression. J Biol Chem. 2010;285:21496-21507.

22. Shi XB, Xue L, Yang J, et al. An androgen-regulated miRNA suppresses Bak1 expression and induces androgen-independent growth of prostate cancer cells. Proc Natl Acad Sci USA. 2007;104:19983-19988.

23. Zhu J, Tang B, Lv X, et al. Transcriptomic aberrations and revealing clinical relevance as diagnostic and prognostic biomarker in Hepatocellular Carcinoma. Front Oncol. 2020;10:519180.

24. Zhu L, Yan D, Chen Y, Chen S, Chen N, Han J. The identification of autophagy-related genes in the prognosis of oral squamous cell carcinoma. Oral Dis. 2020;26:1659-1667.

25. Konishi M, Erdem SS, Weissleder R, Lichtman AH, McCarthy JR, Libby P. Imaging Granzyme B activity assesses immune-mediated myocarditis. Circ Res. 2015;117:502-512.

26. Wu CH, Li J, Li L, et al. Extracellular vesicles derived from natural killer cells use multiple cytotoxic proteins and killing mechanisms to target cancer cells. J Extracell Vesicles. 2019;8:1588538.

27. Salama P, Phillips M, Platell C, Iacopetta B. Low expression of Granzyme B in colorectal cancer is associated with signs of early metastastic invasion. Histopathology. 2011;59:207-215.

28. Chiusolo V, Jacquemin G, Yonca Bassoy E, et al. Granzyme B enters the mitochondria in a Sam50-, Tim22- and mtHsp70dependent manner to induce apoptosis. Cell Death Differ. 2017;24:747-758. 
29. Jacquemin G, Margiotta D, Kasahara A, et al. Granzyme B-induced mitochondrial ROS are required for apoptosis. Cell Death Differ. 2015;22:862-874.

30. Zhou Z, He H, Wang K, et al. Granzyme A from cytotoxic lymphocytes cleaves GSDMB to trigger pyroptosis in target cells. Science. 2020;368:1-15.

31. Kumari P, Russo AJ, Shivcharan S, Rathinam VA. AIM2 in health and disease: inflammasome and beyond. Immunol Rev. 2020;297:83-95.

32. Choubey D. Absent in melanoma 2 proteins in the development of cancer. Cell Mol Life Sci. 2016;73:4383-4395.

33. Fujita T, Reis LF, Watanabe N, Kimura Y, Taniguchi T, Vilcek J. Induction of the transcription factor IRF-1 and interferon-beta mRNAs by cytokines and activators of second-messenger pathways. Proc Natl Acad Sci USA. 1989;86(24):9936-9940.

34. Kamijo R, Harada H, Matsuyama T, et al. Requirement for transcription factor IRF-1 in NO synthase induction in macrophages. Science. 1994;263:1612-1615.

35. Bouker KB, Skaar TC, Riggins RB, et al. Interferon regulatory factor-1 (IRF-1) exhibits tumor suppressor activities in breast cancer associated with caspase activation and induction of apoptosis. Carcinogenesis. 2005;26(9):1527-1535.

36. Man SM, Karki R, Malireddi RK, et al. The transcription factor IRF1 and guanylate-binding proteins target activation of the AIM2 inflammasome by Francisella infection. Nat Immunol. 2015;16(5):467-475.

37. Karki R, Sharma BR, Lee E, et al. Interferon regulatory factor 1 regulates PANoptosis to prevent colorectal cancer. JCI Insight. 2020;5:1-13.

38. Stiewe T. The p53 family in differentiation and tumorigenesis. Nat Rev Cancer. 2007;7(3):165-168.

39. Nekulova M, Holcakova J, Coates P, Vojtesek B. The role of p63 in cancer, stem cells and cancer stem cells. Cell Mol Biol Lett. 2011;16 (2):296-327

40. Gonfloni S, Caputo V, Iannizzotto V. P63 in health and cancer. Int J Dev Biol. 2015;59(1-3):87-93.

41. Pflaum J, Schlosser S, Muller M. p53 family and cellular stress responses in cancer. Front Oncol. 2014;4:285.

42. Conde E, Angulo B, Redondo P, et al. The use of P63 immunohistochemistry for the identification of squamous cell carcinoma of the lung. PLoS One. 2010;5(8):e12209.

43. Sniezek JC, Matheny KE, Westfall MD, Pietenpol JA. Dominant negative p63 isoform expression in head and neck squamous cell carcinoma. Laryngoscope. 2004;114(12):2063-2072.

44. Di Como CJ, Urist MJ, Babayan I, et al. p63 expression profiles in human normal and tumor tissues. Clin Cancer Res. 2002;8 (2):494-501.
45. McKeon FD. P63 and p73 in tumor suppression and promotion. Cancer Res Treat. 2004;36(1):6-12.

46. Somerville TDD, Xu Y, Miyabayashi K, et al. TP63-mediated enhancer reprogramming drives the squamous subtype of pancreatic ductal adenocarcinoma. Cell Rep. 2018;25(7):1741-1755.

47. Feng $X$. Regulatory roles and molecular signaling of TNF family members in osteoclasts. Gene. 2005;350:1-13.

48. Al-Lamki RS, Sadler TJ, Wang J, et al. Tumor necrosis factor receptor expression and signaling in renal cell carcinoma. Am J Pathol. 2010;177:943-954.

49. Croft M, Benedict CA, Ware CF. Clinical targeting of the TNF and TNFR superfamilies. Nat Rev Drug Discov. 2013;12:147-168.

50. McDonald C, Chen FF, Ollendorff V, et al. A role for Erbin in the regulation of Nod2-dependent NF-kappaB signaling. J Biol Chem. 2005;280:40301-40309.

51. Kanneganti TD, Lamkanfi M, Núñez G. Intracellular NOD-like receptors in host defense and disease. Immunity. 2007;27:549-559.

52. Liu Y, Yang H, Liu LX, et al. NOD2 contributes to myocardial ischemia/reperfusion injury by regulating cardiomyocyte apoptosis and inflammation. Life Sci. 2016;149:10-17.

53. Du P, Fan B, Han H, et al. NOD2 promotes renal injury by exacerbating inflammation and podocyte insulin resistance in diabetic nephropathy. Kidney Int. 2013;84:265-276.

54. Fritsch M, Günther SD, Schwarzer R, et al. Caspase- 8 is the molecular switch for apoptosis, necroptosis and pyroptosis. Nature. 2019;575:683-687.

55. Grossman WJ, Verbsky JW, Tollefsen BL, Kemper C, Atkinson JP, Ley TJ. Differential expression of granzymes A and B in human cytotoxic lymphocyte subsets and $\mathrm{T}$ regulatory cells. Blood. 2004;104:2840-2848.

56. Gondek DC, Lu LF, Quezada SA, Sakaguchi S, Noelle RJ. Cutting edge: contact-mediated suppression by CD4+CD25+ regulatory cells involves a granzyme B-dependent, perforin-independent mechanism. J Immunol. 2005;174:1783-1786.

57. Cao X, Cai SF, Fehniger TA, et al. Granzyme B and perforin are important for regulatory $\mathrm{T}$ cell-mediated suppression of tumor clearance. Immunity. 2007;27:635-646.

58. Loebbermann J, Thornton H, Durant L, et al. Regulatory $\mathrm{T}$ cells expressing granzyme B play a critical role in controlling lung inflammation during acute viral infection. Mucosal Immunol. 2012;5:161-172.

59. Saito T, Nishikawa H, Wada H, et al. FOXP3(+)CD4(+) T cell subpopulations distinctly control the prognosis of colorectal cancers. Nat Med. 2016;22:679-684.
International Journal of General Medicine

\section{Publish your work in this journal}

The International Journal of General Medicine is an international, peer-reviewed open-access journal that focuses on general and internal medicine, pathogenesis, epidemiology, diagnosis, monitoring and treatment protocols. The journal is characterized by the rapid reporting of reviews, original research and clinical studies across all disease areas. The manuscript management system is completely online and includes a very quick and fair peer-review system, which is all easy to use. Visit http://www.dovepress.com/ testimonials.php to read real quotes from published authors. 